A. Gibson (Culham) showed, may prove excessively expensive, and may be subject to serious plasma pressure limitations. "Astrons," described by M. Christofilos (Livermore, USA), which use a relativistic proton beam to heat the plasma and to maintain the magnetic field configuration, require the development of a smoke-ring accelerator to provide the necessary $1,000 \mathrm{MW}$ of protons at $4 \mathrm{GeV}$.

All six systems raise broadly similar problems of nuclear engineering. There is a neutron flux out of the plasma comparable with that in a fast breeder reactor which (as discussed by B. Myers of Livermore, USA, and M. T. Robinson of Oak Ridge National Laboratory) will displace every atom in the surrounding niobium wall 100 times per annum and will transmute 10 per cent to zirconium over the twenty year lifetime of the reactor. These neutrons must be used with high efficiency in a surrounding cooling blanket containing lithium to breed tritium (a process shown to be feasible by the calculations reported by $\mathrm{F}$. Blow (Harwell) and D. Steiner (Oak Ridge). This somewhat hazardous substance must be extracted and recirculated into the plasma with negligible loss to the outside world. The helium-4 produced by the fusion reactions must be extracted from the plasma without burning up any wall which it encounters. It was clear that these and other technological problems are all broadly soluble, at a cost. Here again, further work is required to determine which solution is the most economic, and how they affect the choice of confinement system.

Although most of the conference was devoted to severely practical engineering matters, some consideration was given to the long term implications of fusion power when J. L. Tuck (Los Alamos) and A. P. Fraas (Oak Ridge) discussed the "social" advantages of fusion as compared with fission--its relative safety, freedom from thermal pollution, and absence of radioactive wastes - and the conditions in which it could earn a dominant place in a national power programme on economic merit alone. That these two points of view are not necessarily contradictory was emphasized by H. Kronberger, the Member for Reactors of the United Kingdom Atomic Energy Authority, who argued that the "social" andvantages were unlikely to tip any scales in favour of fusion unless or until they acquired a quantifiable economic value.

\section{NUCLEAR TARGETS}

\section{New Polarized Protons}

THe new and efficient technique for lining up the spins of protons with butanol and water seems to bo exceeding expectation. A. D. Hill and colleagues (Phys. Rev. Lett., 23, 460; 1969) have cooled a sample of butanol and water to $0.5 \mathrm{~K}$ and have achieved an unprecedented 67 per cent proton polarization, which compares very favourably with the trend indicated by M. Borghini (Bull. Amer. Phys. Soc., 14, 189; 1969), who found a polarization of about 40 per cent near $1 \mathrm{~K}$.

These results are of particular importance for nuclear scattering experiments. The traditional target for spin oriented scattering experiments, where the spin interaction between a proton and some other particle such as a $\pi$-meson or another proton is under study, is lanthanum magnesium nitrate. This substance has a great deal of water of crystallization-twenty-four molecules in all - and the procedure is to try to polarize as many of the hydrogen atoms as possible. The great advantage of using alcohol mixtures, however, is that there are far more hydrogen atoms present and there is therefore more chance of coaxing a large number of protons into the required spin orientation.

Hill et al. carried out their experiments with a sample containing about 95 per cent butanol, 5 per cent water and 0.75 per cent of the free radical porphyrexide. The sample weighed $0 \cdot 2 \mathrm{~g}$. A practical target for a nuclear experiment would be about $10 \mathrm{~g}$, and they estimate that the helium-3 refrigeration capacity at $0.5 \mathrm{~K}$ for a target of this size should be within the practical range of about $10 \mathrm{~mW}$. The effects of having different amounts of porphyrexide present are largely unknown, and Hill et al. surmise that larger or smaller quantities may produce an even higher polarization.

Although Hill et al. are still groping to find a satisfictory explanation for the rise in proton polarization as the temperature of the sample is lowered, they suggest that a model which includes electron spin-spin effects should be able to predict the right sort of temperature variation. In particular they mention the "cross-relaxation" model which would give just the required variation. But the theoretical basis for the models is still too insecure to predict much more than that the polarization of the sample will continue increasing-with diminishing returns-as the temperature of the sample is lowered.

The work was carried out at the Argonne National Laboratory, and investigations on a similar line are also in progress at Brookhaven and at CERN. The first scattering experiment actually to use a butanol and water target was reported at a recent conference in New York.

\section{CYCLOTRONS \\ New Mileage from Accelerutors}

\section{from a Correspondent}

THE development of the compact cyclotron, interest in computer control and the growing use of the instruments for experiments other than nuclear physics have marked a now phase in cyclotron development. This was the message of W. Marshall (Atomic Energy Research Establishment, Harwell) when he opened the fifth international cyclotron conference held in Oxford from September 17 to 19 .

Reviewing the innovations in isochronous cyclotrons, J. A. Martin (Oak Ridge National Laboratory) said that great flexibility would be given by the separated sector cyclotrons. Three major projects of this type have been approved, the $585 \mathrm{MeV}$ cyclotron of the Swiss Institute for Nuclear Research to be built near Zurich, the $500 \mathrm{MeV} \mathrm{H}$ - cyclotron of the Tri-University Meson Facility at Vancouver and the $200 \mathrm{MeV}$ variable energy, multiparticle cyclotron of Indiana University. The most important recent development has been a great upsurge in interest in accelerating very heavy ions for producing super heavy nuclei with atomic numbers near 114. Among the suggested reactions are the bombardment of uranium with uranium and lead with lead at energies up to $10 \mathrm{MeV} /$ nucleon.

Many new high energy, very heavy ion cyclotrons have been proposed for the United States. Most have an electrostatic accelerator as the injector and strip 